
2004 Kluwer Academic Publishers. Manufactured in The Netherlands.

\title{
Supernormal Vector Configurations
}

\author{
SERKAN HOŞTEN \\ serkan@math.sfsu.edu \\ Department of Mathematics, San Francisco State University, San Francisco, CA 94132, USA
}

DIANE MACLAGAN*

maclagan@math.stanford.edu

Department of Mathematics, Stanford University, Stanford, CA 94305, USA

bernd@math.berkeley.edu

BERND STURMFELS ${ }^{\dagger}$

Department of Mathematics, University of California, Berkeley, CA 94720, USA

Received May 7, 2001; Revised May 29, 2003

Abstract. A configuration of lattice vectors is supernormal if it contains a Hilbert basis for every pointed cone spanned by a subset. We study such configurations from various perspectives, including triangulations, integer programming and Gröbner bases. Our main result is a bijection between virtual chambers of the configuration and virtual initial ideals of the associated binomial ideal.

Keywords: triangulation, chamber complex, initial idea, Groebner fan

\section{Introduction}

Let $B=\left\{b_{1}, \ldots, b_{n}\right\} \subseteq \mathbb{Z}^{m}$ and let cone $(B)$ be the polyhedral cone in $\mathbb{R}^{m}$ spanned by $B$. We assume that $B$ does not contain the zero vector or a vector that is a positive multiple of another vector. The configuration $B$ is normal if every lattice point in $\operatorname{cone}(B)$ is a nonnegative integer combination of $B$. We say that $B$ is supernormal if, for every subset $B^{\prime}$ of $B$, every lattice point in cone $\left(B^{\prime}\right)$ is a non-negative integer combination of $B \cap \operatorname{cone}\left(B^{\prime}\right)$.

In Section 2 we discuss supernormal configurations in low dimensions. In particular, we exhibit a finitely generated submonoid of $\mathbb{Z}^{3}$ which cannot be generated by a finite supernormal subset. This implies that in general the process of normalization [8, Algorithm 13.2] cannot be extended to produce a finite supernormal generating set.

In Section 3 we characterize supernormal vector configurations in terms of polyhedral geometry (triangulations) and in terms of integer programming (total dual integrality). This will generalize the familiar characterizations of unimodular configurations [8, Section 8]. Recall that a configuration $B$ in $\mathbb{Z}^{m}$ is unimodular if, for every subset $B^{\prime}$ of $B$, every lattice point in cone $\left(B^{\prime}\right)$ is a non-negative integer combination of $B^{\prime}$. Supernormal configurations thus lie between unimodular and normal configurations; every unimodular configuration is supernormal, and every supernormal configuration is normal.

*Supported by NSF grant DMS 97-29992 through IAS Princeton.

${ }^{\dagger}$ Supported by NSF grant DMS 99-70254. 
The algebraic theory of integer programming is closely related to Gröbner bases of binomial ideals [9]. We encode our configuration $B$ as the ideal $J_{B}$ in the polynomial ring $k\left[x_{1}, \ldots, x_{n}\right]$ generated by

$$
\prod_{i: u \cdot b_{i}>0} x_{i}^{u \cdot b_{i}}-\prod_{j: u \cdot b_{j}<0} x_{j}^{-u \cdot b_{j}} \quad \text { where } u \text { runs over } \mathbb{Z}^{m} .
$$

In the language of [4] or [6, Section 3.3], the ideal $J_{B}$ is the lattice ideal for the lattice spanned by the rows of the $(m \times n)$-matrix $\left(b_{1}, \ldots, b_{n}\right)$.

Every vector $w \in \operatorname{cone}(B)$ defines an initial ideal of $J_{B}$ as follows: $i n_{w}\left(J_{B}\right)$ is generated by the monomials $\prod_{i: u \cdot b_{i}>0} x_{i}^{u \cdot b_{i}}$ where $u \in \mathbb{Z}^{m}$ satisfies $u \cdot w>0$ and the binomials of the form in Eq. (1) where $u \in \mathbb{Z}^{m}$ satisfies $u \cdot w=0$. Two vectors $w, w^{\prime} \in \operatorname{cone}(B)$ lie in the same cell of the Gröbner fan of $J_{B}$ if $i n_{w}\left(J_{B}\right)=i n_{w^{\prime}}\left(J_{B}\right)$, and they lie in the same cell of the chamber complex of $B$ if, for every subset $B^{\prime}$ of $B, w \in \operatorname{cone}\left(B^{\prime}\right)$ if and only if $w^{\prime} \in \operatorname{cone}\left(B^{\prime}\right)$. In Section 4 we prove:

Theorem 1.1 If the configuration $B$ is supernormal then the chamber complex of $B$ coincides with the Gröbner fan of $J_{B}$.

We note that the converse statement does not hold, even for $m=1$. For the special case when $B$ is unimodular, this theorem follows from [8, Proposition 8.15] via Gale duality. Our proof will be self-contained.

A longstanding conjecture [9] states that the number of facets of any chamber in the Gröbner fan of $J_{B}$ is bounded by a function of $m$ alone, independent of the coordinates of the $b_{i}$. In Section 5 we examine this question for the supernormal configuration

$$
B=\left\{(1, u, v) \in \mathbb{Z}^{3}:(u, v) \in P \cap \mathbb{Z}^{2}\right\}
$$

associated with a convex lattice polygon $P$ in the plane. The chamber complex of $B$ is gotten by drawing the line segments connecting any two lattice points in $P$ as in figure 1 . It is an open question whether polygons with arbitrarily many edges can appear in such a picture. See Proposition 5.4 for the current status of the problem.

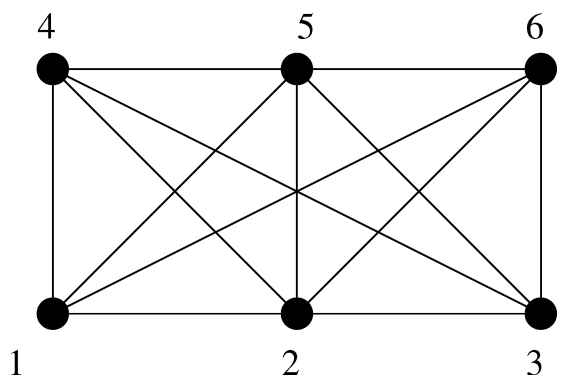

Figure 1. Chamber complex of a rectangle. 
The chambers of a vector configuration $B$ are in bijection with the regular triangulations of a Gale dual configuration $A$. This was extended in [3] to a bijection between all triangulations of $A$ and virtual chambers of $B$. We reexamine these concepts in Section 6 , and we introduce the following algebraic analogue: A monomial ideal $M$ in $k\left[x_{1}, \ldots, x_{n}\right]$ is a virtual initial ideal of $J_{B}$ if $M$ has the same Hilbert function as $J_{B}$ with respect to the finest grading which makes $J_{B}$ homogeneous. In [8, Section 10] such $M$ were called $A$-graded monomial ideals. There is a map from virtual initial ideals to virtual chambers (defined by [8, Theorem 10.10]) but this map is in general neither injective nor surjective. Our main result is the following extension of Theorem 1.1.

Theorem 1.2 If the configuration B is supernormal then the map from virtual initial ideals of $J_{B}$ to virtual chambers of $B$ is a bijection.

In $A$-graded language this has the following formulation.

Corollary 1.3 If A is a matrix whose Gale dual B is supernormal, then there is a bijection between monomial A-graded ideals and triangulations of $A$.

\section{Examples and counterexamples}

In this section we study examples of supernormal configurations in low dimensions. Recall that a configuration $B$ of vectors in $\mathbb{Z}^{m}$ is normal if it generates the monoid $\mathbb{Z}^{m} \cap$ cone $(B)$. We call $B$ pointed if there exists $u \in \mathbb{R}^{m}$ such that $b_{i} \cdot u>0$ for all $i$. We say that $B$ is a Hilbert basis if $B$ is pointed and minimally generates the monoid $\mathbb{Z}^{m} \cap \operatorname{cone}(B)$. Clearly, if $B$ is a Hilbert basis then $B$ is normal.

Dimension one: If $m=1$ then $B$ consists of a single vector, say $b$, or of the form $\left\{b_{1}, b_{2}\right\}$ where $b_{1}<0<b_{2}$. The configuration $B$ is normal if and only if either $b=1$, or $b=-1$, or $b_{1}<0, b_{2}>0$ and $\operatorname{gcd}\left(b_{1}, b_{2}\right)=1$. But $B$ is supernormal if and only if either $b=1$, or $b=-1$, or $B=\{-1,+1\}$. Thus $B=\{-2,3\}$ is normal but not supernormal. We conclude that a one dimensional pointed configuration is normal if and only if it is supernormal.

The chamber complex of $B$ consists of either one or two cones, and it coincides with the Gröbner fan of the principal ideal $J_{B}$. For instance, for $B=\{-2,3\}$ the ideal $J_{B}=\left\langle x^{2}-y^{3}\right\rangle$ has two initial ideals, but for $B=\{2,3\}$ we get $J_{B}=\left\langle x^{2} y^{3}-1\right\rangle$ which has only one initial ideal. This shows that the converse to Theorem 1.1 does not hold.

Dimension two: The configuration $B$ consists of distinct nonzero vectors in $\mathbb{Z}^{2}$. We assume that their ordering $b_{1}, b_{2}, \ldots, b_{n}$ is counterclockwise, with $b_{1}$ and $b_{n}$ being the extreme rays if $B$ is pointed, and we set $b_{n+1}=b_{1}$ otherwise. They lie in an open half-plane if and only if $B$ is pointed. The last statement from the $m=1$ case does not hold for $m=2$ : the configuration $B=\{(1,0),(1,2),(0,1)\}$ is pointed and normal but not supernormal.

Proposition 2.1 A configuration $B=\left\{b_{1}, \ldots, b_{n}\right\} \subseteq \mathbb{Z}^{2}$ is supernormal if and only if $\operatorname{det}\left(b_{i}, b_{i+1}\right)=1$ for $i=1,2, \ldots, n-1$, and also $\operatorname{det}\left(b_{n}, b_{1}\right)=1$ if $B$ is not pointed. 
Proof: Suppose $B$ is supernormal. Note that cone $\left(b_{i}, b_{i+1}\right)$ contains no other $b_{j}$, so $b_{i}$ and $b_{i+1}$ must be a Hilbert basis for cone $\left(b_{i}, b_{i+1}\right) \cap \mathbb{Z}^{2}$, and thus we have $\operatorname{det}\left(b_{i}, b_{i+1}\right)=1$.

Conversely, suppose that $\operatorname{det}\left(b_{i}, b_{i+1}\right)=1$ for all $i$. This means that any lattice point in cone $\left(b_{i}, b_{i+1}\right)$ can be written as a non-negative integer combination of $b_{i}$ and $b_{i+1}$. Every cone generated by a subset $B^{\prime}$ of the $b_{i}$ can be decomposed as a union of cones of the form cone $\left(b_{i}, b_{i+1}\right)$, so our assumption implies that every lattice point in cone $\left(B^{\prime}\right)$ can be written as a non-negative integer combination of the vectors in $B \cap$ cone $\left(B^{\prime}\right)$. Therefore $B$ is supernormal.

Corollary 2.2 Every two-dimensional Hilbert basis is supernormal.

In the language of algebraic geometry, Proposition 2.1 says that $B \subseteq \mathbb{Z}^{2}$ is supernormal if and only if the toric surface $X_{\Delta}$ is smooth, where $\Delta$ is the fan whose rays are the vectors in $B$. In higher dimensions, supernormality means that all toric varieties that share a fixed Cox homogeneous coordinate ring are smooth. This follows from Proposition 3.1 below.

Dimension three: Corollary 2.2 does not hold for $m=3$. Take

$$
B=\{(1,0,0),(0,1,0),(1,1,1),(1,1,2),(1,2,3),(1,2,4)\} \subseteq \mathbb{Z}^{3}
$$

This is the Hilbert basis for the cone spanned by $(1,0,0),(0,1,0)$ and $(1,2,4)$. The configuration $B$ is not supernormal. To see this consider $B^{\prime}=\{(0,1,0),(1,1,1),(1,2,3)\}$ and note that $(1,2,2)$ lies in cone $\left(B^{\prime}\right) \cap \mathbb{Z}^{3}$ but not in the monoid generated by cone $\left(B^{\prime}\right) \cap B=B^{\prime}$. If we add the vector $(1,2,2)$ to $B$ then the resulting configuration of seven vectors is supernormal.

It is well-known that the monoid of lattice points in any pointed rational polyhedral cone has a finite Hilbert basis. In the previous example, the Hilbert basis can be enlarged to a finite supernormal generating set. This raises the question of whether every rational submonoid of $\mathbb{Z}^{m}$ is generated by a finite supernormal subset. This is not the case.

Theorem 2.3 The monoid of lattice points in the three-dimensional cone spanned by $P_{0}=(-1,1,2), P_{1}=(1,-1,1), P_{2}=(0,1,0)$ and $P_{3}=(1,0,0)$ is not generated by $a$ finite supernormal subset.

Proof: Since $P_{0}, P_{1}, P_{2}$ and $P_{3}$ are the first lattice points on the extreme rays of this cone, any supernormal generating set must contain these vectors. Consider the following sequence of vectors in this monoid:

$$
P_{i}:=\frac{1}{2} \cdot\left(P_{i-2}+P_{i-1}+P_{i^{\prime}}\right) \text { for } i \geq 4
$$

where $i^{\prime}=(i \bmod 2)$. Explicitly,

$$
P_{2 i}=(0,1, i-1), \quad P_{2 i+1}=(1,0, i-1) \quad \text { for } i \geq 1
$$


At each stage in this iteration, the three vectors $P_{i-2}, P_{i-1}, P_{i^{\prime}}$ generate an index two sublattice of $\mathbb{Z}^{3}$, and $P_{i}$ is the unique vector which completes the Hilbert basis for their triangular cone. Suppose there is a finite supernormal generating set $B$ for the ambient monoid and consider the smallest index $i$ such that $P_{i}$ is not in $B$. Then the subset $B^{\prime}=$ $\left\{P_{i-2}, P_{i-1}, P_{i^{\prime}}\right\}$ violates the defining property of $B$ being supernormal.

While this result shows that not every configuration can be embedded into a supernormal one, there do exist interesting specific supernormal configurations in higher dimensions, beyond the familiar class of unimodular configurations. Here is an example for $m=3$ :

Example 2.4 The configuration $B=\{-1,0,+1\}^{3}$ of all 27 vectors whose coordinates have absolute value at most one is supernormal.

A configuration $B$ is called convex if it is gotten from the set of all lattice points in a convex polytope $P$ by prepending an extra coordinate " 1 ". Thus (2) is a convex configuration in dimension three. The three dimensional convex configurations arising from convex polygons $P$ play a special role and are discussed in detail in Section 5. In Proposition 5.1 we show that they are supernormal.

Dimension four and beyond: Most convex configurations in higher dimensions are not supernormal, however. Consider the cone over the three-dimensional cube given by the columns of

$$
\left(\begin{array}{llllllll}
1 & 1 & 1 & 1 & 1 & 1 & 1 & 1 \\
0 & 1 & 0 & 1 & 0 & 1 & 0 & 1 \\
0 & 0 & 1 & 1 & 0 & 0 & 1 & 1 \\
0 & 0 & 0 & 0 & 1 & 1 & 1 & 1
\end{array}\right) .
$$

This configuration of eight vectors in $\mathbb{Z}^{4}$ is convex but not supernormal. What is missing is the vector $(2,1,1,1)$ which represents the centroid of the cube. The configuration together with $(2,1,1,1)$ is supernormal.

It would be interesting to identify infinite families of configurations in higher dimensions which are supernormal but not unimodular. Such families might arise from graph theory or combinatorial optimization.

\section{Polyhedral characterizations}

In this section we present two characterizations of supernormal configurations $B$. The first is in terms of triangulations, and the second involves the concept of total dual integrality from integer programming.

A subdivision of a vector configuration $B=\left\{b_{1}, \ldots, b_{n}\right\} \subseteq \mathbb{Z}^{m}$ is a polyhedral fan $\Delta$ in $\mathbb{R}^{m}$ whose support is $\operatorname{cone}(B)$ and each of whose rays is spanned by a vector $b_{i}[10$, Section 9]. It is customary to identify $\Delta$ with the collection of subsets $\sigma$ of $B$ which lie in the maximal cones of $\Delta$. A subdivision $\Delta$ is regular if there exists a vector $c \in \mathbb{Z}^{n}$ such 
that $\sigma \subseteq B$ is a face of $\Delta$ if and only if there exists an $x \in \mathbb{Z}^{m}$ with $b_{i} \cdot x=c_{i}$ for all $b_{i} \in \sigma$ and $b_{i} \cdot x<c_{i}$ otherwise. Every choice of vector $c$ gives rise to a subdivision of $B$. A subdivision $\Delta$ of $B$ is a triangulation if each maximal cell $\sigma$ has precisely $m$ elements. A triangulation $\Delta$ of $B$ is unimodular if every maximal cell $\sigma$ is a lattice basis for $\mathbb{Z}^{m}$. The triangulation $\Delta$ uses all vectors if each element $b_{i}$ of $B$ spans a ray of the fan $\Delta$.

A configuration $B$ is unimodular if and only if every triangulation of $B$ is unimodular. Here it suffices to consider regular triangulations. We prove an analogous characterization for supernormal configurations.

Proposition 3.1 For a configuration $B$, the following are equivalent:

1. B is supernormal.

2. Every triangulation of $B$ that uses all vectors is unimodular.

3. Every regular triangulation of $B$ that uses all vectors is unimodular.

Proof: We first prove (1) $\Rightarrow$ (2). Let $B$ be supernormal, $\Delta$ a triangulation that uses all vectors, and $\sigma=\left\{b_{i_{1}}, \ldots, b_{i_{m}}\right\}$ a maximal cell of $\Delta$. If $\sigma$ is not a lattice basis of $\mathbb{Z}^{m}$ then $\sigma$ does not generate the monoid $\mathbb{Z}^{m} \cap$ cone $(\sigma)$. Supernormality implies that cone $(\sigma)$ contains at least one other vector $b_{j} \in B \backslash \sigma$, but then this vector $b_{j}$ cannot be used in the triangulation $\Delta$. This contradicts our hypothesis.

The implication (2) $\Rightarrow$ (3) is trivial. It remains to show (3) $\Rightarrow$ (1). Suppose (3) holds. Let $B^{\prime}$ be any subset of $B$. We construct a regular subdivision of $B$ which has $\sigma=B \cap \operatorname{cone}\left(B^{\prime}\right)$ as one of its faces, and which uses all vectors in $B \backslash \sigma$ as rays. To do this, we use a vector $c$ which has $c_{i}=0$ for $i \in \sigma$, and $c_{i}>0$ for $i \notin \sigma$, choosing the positive coordinates inductively so as to ensure that all vectors of $B \backslash \sigma$ appear. This subdivision can be refined to a regular triangulation $\Delta$ of $B$ that uses all vectors. By hypothesis, $\Delta$ is unimodular, and its restriction to $\sigma$ is a unimodular triangulation of $\sigma$. This implies that $\sigma$ generates the monoid cone $\left(B^{\prime}\right) \cap \mathbb{Z}^{m}$. We conclude that $B$ is supernormal.

Regular subdivisions are polar to the polyhedra with facet normals in $B=\left\{b_{1}, \ldots, b_{n}\right\}$. More precisely, for $c \in \mathbb{Z}^{n}$ we define the polyhedron

$$
P_{c}=\left\{x \in \mathbb{R}^{m}: b_{i} \cdot x \leq c_{i} \text { for } i=1, \ldots, n\right\} .
$$

Let $\mathcal{N}\left(P_{c}\right)$ denote the normal fan of the convex polyhedron $P_{c}$.

Lemma 3.2 The normal fan $\mathcal{N}\left(P_{c}\right)$ is a regular subdivision of $B$. Every regular subdivision of $B$ is the normal fan of $P_{c}$ for some $c \in \mathbb{Z}^{n}$.

Proof: These statements follow from the fact that $\mathcal{N}\left(P_{c}\right)$ is the regular subdivision induced by the vector $c$.

We recall the following definition from integer programming. A good reference for these topics is Chapter 22 of Schrijver's book [7]. 
Definition 3.3 A system of rational inequalities $D x \leq d$ is called totally dual integral (TDI) if for each $w \in \mathbb{Z}^{m}$ such that the linear program $\max \{w \cdot x: D x \leq d\}$ has a finite optimal solution, the dual linear program $\min \{y \cdot d: y D=d, y \geq 0\}$ has an integral solution.

The property of being TDI is a property of the given representation of a polyhedron in terms of inequalities, and not of the polyhedron itself. In what follows, whenever we say "the polyhedron $P_{c}$ is TDI", what we mean is that the inequality system $b_{i} \cdot x \leq c_{i}, i=1, \ldots, n$ is TDI. The following characterization of unimodular configurations is easily derived from the basic properties of TDI systems [7, Section 22].

Proposition 3.4 The vector configuration $B=\left\{b_{1}, \ldots, b_{n}\right\} \subseteq \mathbb{Z}^{m}$ is unimodular if and only if the polyhedron $P_{c}$ is TDI for every $c \in \mathbb{Z}^{n}$.

We will prove an analogous result for supernormal configurations by considering only those polyhedra $P_{c}$ where $c$ ranges over a certain subset of $\mathbb{Z}^{n}$. First we give a name to these special polyhedra.

Definition 3.5 The system of inequalities defining $P_{c}$ is tight if $P_{c-e_{i}} \cap \mathbb{Z}^{m}$ is strictly contained in $P_{c} \cap \mathbb{Z}^{m}$ for every unit vector $e_{i} \in \mathbb{Z}^{n}$.

Tightness is a property not of the polyhedron $P_{c}$ but of the inequality system $b_{i} \cdot x \leq$ $c_{i}, \quad i=1, \ldots, n$. However, as with TDI, we shall abuse language by simply saying " $P_{c}$ is tight". With this convention, $P_{c}$ is tight if and only if, for each $i=1, \ldots, n$, there exists a lattice point $x \in P_{c}$ with $b_{i} \cdot x=c_{i}$.

Theorem 3.6 The vector configuration $B=\left\{b_{1}, \ldots, b_{n}\right\} \subseteq \mathbb{Z}^{m}$ is supernormal if and only if every tight polyhedron $P_{c}$ is TDI.

Proof: We first prove the if direction using condition (3) in Proposition 3.1. Let $\Delta$ be a regular triangulation of $B$ which uses all vectors. We wish to show that $\Delta$ is a unimodular triangulation. By Lemma 3.2 there is a simple polyhedron $P_{c}$ whose normal fan equals $\Delta$. In particular, every vector $b_{i}$ defines a facet of $P_{c}$. Since $P_{c}$ is a rational polyhedron, there is some $r>0$ such that $P_{r c}=r P_{c}$ is integral. The polyhedron $P_{r c}$ has normal fan $\Delta$, and is tight, and so is TDI by assumption. Theorem 22.5 in [7] implies that every set $\sigma$ of $m$ vectors in $B$ that define a vertex of $P_{r c}$ is a basis of $\mathbb{Z}^{m}$. These cones $\sigma$ are the maximal cells of $\Delta$. Hence $\Delta$ is unimodular and we conclude that $B$ is supernormal.

For the only-if direction, suppose that $B$ is supernormal and let $c \in \mathbb{Z}^{n}$ be such that $P_{c}$ is tight. Consider any face $F$ of $P_{c}$, and let $\sigma$ be the set of all vectors $b_{i} \in B$ such $b_{i} \cdot x=c_{i}$ holds for all $x \in F$. In view of [7, Theorem 22.5], it suffices to prove that $\sigma$ is a Hilbert basis. Suppose this is not true. Supernormality implies that cone $(\sigma)$ contains at least one other vector $b_{j} \in B \backslash \sigma$. Because $b_{j}$ lies in cone $(\sigma)$, we can write $b_{j}=\sum_{b_{i} \in \sigma} \lambda_{i} b_{i}$ where $\lambda_{i} \geq 0$. Since $P_{c}$ is tight there exists a lattice point $z \in P_{c}$ with $b_{j} \cdot z=c_{j}$. However since $j \notin \sigma$, we know that there is some $x \in F$ for which $b_{j} \cdot x<c_{j}$. The first of these two statements implies $c_{j}=b_{j} \cdot z=\sum_{b_{i} \in \sigma} \lambda_{i}\left(b_{i} \cdot z\right) \leq \sum_{b_{i} \in \sigma} \lambda_{i} c_{i}$. The second implies 
$c_{j}>b_{j} \cdot x=\sum_{b_{i} \in \sigma} \lambda_{i}\left(b_{i} \cdot x\right)=\sum_{b_{i} \in \sigma} \lambda_{i} c_{i}$. But these two statements contradict each other, and so we conclude that $b_{j}$ does not exist, and thus $\sigma$ is a Hilbert basis. It follows that $P_{c}$ is TDI.

\section{Chambers and initial ideals}

The goal of this section is to prove Theorem 1.1 which states that the chamber complex equals the Gröbner fan if $B$ is supernormal. We start out by characterizing these two fans by means of the polyhedra $P_{c}$. In the next two lemmas, $B$ is an arbitrary configuration in $\mathbb{Z}^{m}$.

Lemma 4.1 The chamber complex of $B=\left\{b_{1}, \ldots, b_{n}\right\} \subseteq \mathbb{Z}^{m}$ is the common refinement of the normal fans $\mathcal{N}\left(P_{c}\right)$ as c runs over $\mathbb{Z}^{n}$.

Proof: According to the definition given in the introduction, two vectors lie in the same cell of the chamber complex if and only if they lie in exactly the same cones spanned by linearly independent $m$-subsets of $B$. This holds if and only if, for every regular subdivision $\Delta$ of $B$, they lie in the same cell of $\Delta$. Lemma 3.2 completes the proof.

Lemma 4.1 coincides with the first statement in [6, Proposition 3.3.5]. The term secondary fan is often used for the chamber complex. For $c \in \mathbb{Z}^{n}$ consider the lattice polyhedron

$$
Q_{c}=\operatorname{conv}\left\{x \in \mathbb{Z}^{m}: b_{i} \cdot x \leq c_{i} \text { for } i=1, \ldots, n\right\} .
$$

This is the convex hull of all lattice points in the polyhedron $P_{c}$.

Lemma 4.2 The Gröbner fan of the binomial ideal $J_{B} \subseteq k\left[x_{1}, \ldots, x_{n}\right]$ is the common refinement of the normal fans $\mathcal{N}\left(Q_{c}\right)$ as c runs over $\mathbb{Z}^{n}$.

Proof: This is the second statement of [6, Proposition 3.3.5].

The recipe in the introduction (following Eq. (1)) shows how to derive the initial ideal $i_{w}\left(J_{B}\right)$ associated with a vector $w \in \operatorname{cone}(B)$. Note the following subtlety in our notation: while $w$ is a vector with $m$ coordinates, it specifies a term order on monomials in $n$ variables.

Since $P_{c}$ is a rational polyhedron there is a positive integer $r$ such that $r P_{c}=P_{r c}$ has integer vertices. Hence $\mathcal{N}\left(P_{c}\right)=\mathcal{N}\left(P_{r c}\right)=\mathcal{N}\left(Q_{r c}\right)$. This proves the following well-known result:

Corollary 4.3 For any configuration $B=\left\{b_{1}, \ldots, b_{n}\right\} \subseteq \mathbb{Z}^{m}$, the Gröbner fan of the ideal $J_{B}$ refines the chamber complex of $B$.

This says that the cones in the chamber complex of $B$ can split into smaller cones as one passes to the Gröbner fan of $J_{B}$. It is known that no splitting happens when $B$ is a unimodular configuration; see for example [8, Proposition 8.15(a)]. Theorem 1.1 says that 
no splitting happens even when $B$ is only supernormal. To prove this we need one more lemma:

Lemma 4.4 [7, Corollary 22.1c] If $P_{c}$ is TDI then $P_{c}=Q_{c}$.

Proof of Theorem 1.1: $\quad$ Let $B$ be supernormal. In view of Lemmas 4.1 and 4.2, it suffices to prove the following statement: for any $c \in \mathbb{Z}^{n}$ there exists $c^{\prime} \in \mathbb{Z}^{n}$ such that the normal fan $\mathcal{N}\left(Q_{c}\right)$ of the integral polyhedron $Q_{c}$ equals the normal fan $\mathcal{N}\left(P_{c^{\prime}}\right)$ of the rational polyhedron $P_{c^{\prime}}$. This is done by "pushing in" all facets of $P_{c}$ that do not contain integral points. More precisely, given $c \in \mathbb{Z}^{n}$, let $x^{u}$ be the common divisor of all monomials $x^{c-B z}$ for $z \in Q_{c}$. If $x^{u}=1$, then $P_{c}$ is tight. Otherwise, $P_{c-u}$ is tight and $Q_{c}=Q_{c-u}$. Set $c^{\prime}=c-u$. Since $P_{c^{\prime}}$ is tight, we have that $P_{c^{\prime}}$ is TDI by Theorem 3.6. Using Lemma 4.4, we conclude that $P_{c^{\prime}}=Q_{c^{\prime}}=Q_{c}$ and hence $\mathcal{N}\left(Q_{c}\right)=\mathcal{N}\left(P_{c^{\prime}}\right)$.

\section{How to subdivide a polygon}

Let $P$ be a planar convex polygon with integral vertices. In this section we study convex vector configurations of the following form:

$$
B=\left\{(1, u, v) \in \mathbb{Z}^{3}:(u, v) \in P \cap \mathbb{Z}^{2}\right\} .
$$

We first show that they are all supernormal.

Proposition 5.1 Every convex configuration in $\mathbb{Z}^{3}$ is supernormal.

Proof: Let $B$ be a convex configuration in $\mathbb{Z}^{3}$ and consider any triangulation $\Delta$ of $B$ that uses all vectors. Now a lattice triangle in the plane which contains no other lattice point has area one half (by Pick's theorem, for example). This implies that the triangulation $\Delta$ is unimodular, and so Proposition 3.1 implies that $B$ is supernormal.

The chamber complex of the polygon $P$ is the common refinement of all lattice triangulations of $P$. Hence the chamber complex of the vector configuration $B$ is simply the cone over the chamber complex of $P$. We draw the chamber complex of $P$ by connecting any pair of lattice points in $P$ by a straight line segment.

For example, if $P$ is the quadrangle with vertices $(1,0),(0,1),(2,3)$, and $(3,1)$, then $B$ is the set of column vectors of the $3 \times 8$-matrix:

$$
\left(\begin{array}{llllllll}
1 & 1 & 1 & 1 & 1 & 1 & 1 & 1 \\
1 & 0 & 1 & 2 & 3 & 1 & 2 & 2 \\
0 & 1 & 1 & 1 & 1 & 2 & 2 & 3
\end{array}\right) .
$$

The chamber complex of $P$ is the subdivision of $P$ into 26 triangles, five quadrilaterals, and one pentagon, which is depicted in figure 2 . 


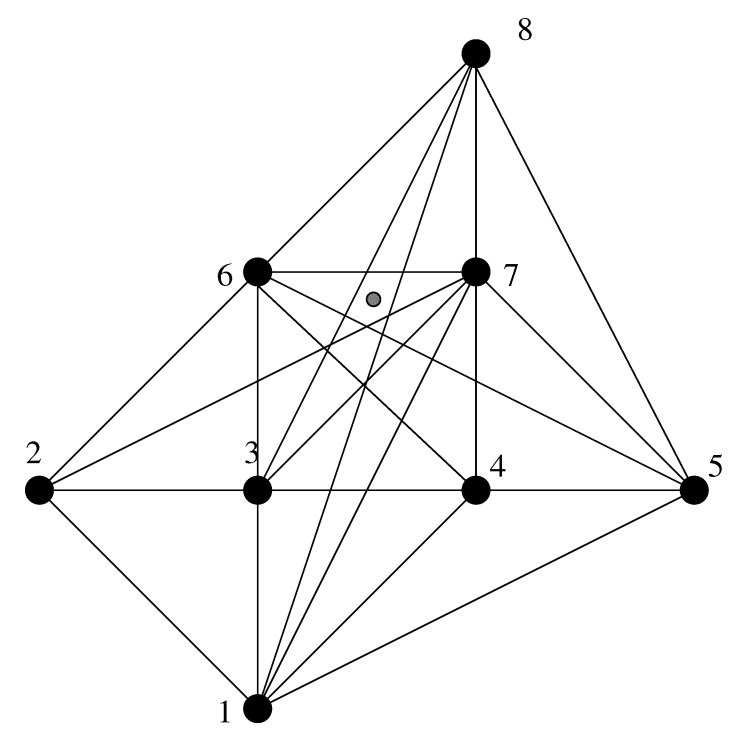

Figure 2. Chamber complex with a pentagonal chamber.

We write $\mu(P)$ for the maximum number of edges of any region in the chamber complex of a lattice polygon $P$. For instance, in figure 2 we have $\mu(P)=5$. The main point of this section is the open question of whether there exists a global upper bound for the numbers $\mu(P)$.

Problem 5.2 (The Polygon Problem) Does there exist a constant $N$ such that every convex lattice polygon $P$ satisfies $\mu(P) \leq N$ ?

We circulated this problem in October 2000, and in the meantime considerable progress has been made by several people. However, the problem remains open for now. Later in this section we will summarize what is known at the present time (April 2001).

The Polygon Problem is important to us because it is a special case of a conjecture in the algebraic theory of integer programming. Sturmfels and Thomas [9, Conjecture 6.1] asked whether there exists a finite bound $\phi(m)$ on the number of facets of any cone in the Gröbner fan of an ideal $J_{B}$ having codimension $m$. Such a bound would have implications for the sensitivity analysis of integer programming in fixed dimension $m$. It is obvious that $\phi(2)=2$, and it was conjectured in [9, Conjecture 6.2] that $\phi(3)=4$. The latter conjecture was much too optimistic. It is now easily seen to be false: Figure 2 together with the following proposition implies $\phi(3) \geq 5$ :

Proposition 5.3 Every lattice polygon $P$ satisfies $\phi(3) \geq \mu(P)$.

Proof: The chamber complex of a supernormal configuration $B$ is the Gröbner fan of the associated binomial ideal $J_{B}$. Hence $\phi(m)$ is greater or equal to the maximum number 
of facets of any cone in the chamber complex of a supernormal configuration in $\mathbb{Z}^{m}$. For $m=3$ we can take the chamber complex of a polygon $P$ to get a lower bound for $\phi(3)$.

The first counterexamples to [9, Conjecture 6.2] were given by Hoşten and Maclagan [5] who showed that $\phi(3) \geq 6$. However, the question of whether $\phi(3)$ is finite remains open. A negative answer to the Polygon Problem would show that $\phi(m)$ is infinite for $m \geq 3$.

To illustrate our algebraic interpretation of planar chamber complexes, we translate the marked pentagonal chamber in figure 2 into a specific reduced Gröbner basis of binomials. Our ideal is generated by the three binomials corresponding to the rows of the matrix in (3):

$$
J_{B}=\left\langle x_{1} x_{2} x_{3} x_{4} x_{5} x_{6} x_{7} x_{8}-1, x_{1} x_{3} x_{4}^{2} x_{5}^{3} x_{6} x_{7}^{2} x_{8}^{2}-1, x_{2} x_{3} x_{4} x_{5} x_{6}^{2} x_{7}^{2} x_{8}^{3}-1\right\rangle .
$$

We next fix a term order which refines any non-negative real weight vector $\left(u_{1}, \ldots, u_{8}\right)$ with the property that $w=\sum_{i=1}^{8} u_{i} b_{i}$ lies in the marked pentagonal chamber of $B=$ $\left\{b_{1}, \ldots, b_{8}\right\} \in \mathbb{Z}^{3}$. For instance, we can take $u=(0,0,0,0,1,4,1,0)$. The reduced Gröbner basis of $J_{B}$ with respect to this term order equals:

$$
\begin{aligned}
& \frac{x_{2}^{4} x_{3} x_{6}^{2}-x_{4}^{2} x_{5}^{5} x_{7}}{x_{6} x_{8}^{2}-x_{1}^{3} x_{3} x_{4}^{2} x_{5}^{3}}, x_{5} x_{7} x_{8}^{2}-x_{1}^{2} x_{2}^{2} x_{3}^{2} x_{7} x_{8}-x_{2} x_{6} x_{8}-x_{1} x_{2}^{2} x_{3} x_{6} x_{5}^{2}, x_{5}, \underline{x_{7} x_{8}^{3}-x_{1}^{2} x_{5}^{2} x_{5}^{3} x_{7}-x_{2}^{2} x_{6}}, \\
& \left.\quad \underline{x_{1}^{2} x_{2} x_{3} x_{4} x_{5}-x_{8}}, \quad x_{1}^{2} x_{3} x_{4}^{2} x_{5}^{3} x_{7}-1\right\}
\end{aligned}
$$

The five "flippable" Gröbner basis elements are underlined. They correspond to the five edges of the pentagonal chamber in figure 2 .

We shall now present what is known on the Polygon Problem. The following result is an outgrowth of the combined efforts of Miguel Azaola, Jesús de Loera, Jörg Rambau, Francisco Santos, Marc Pfetsch and Günter Ziegler. In November 2000, the first four of these obtained the lower bound of 12 . It is attained by the $8 \times 84$ lattice rectangle. In April 2001, the last two succeeded in improving the previous world record ${ }^{1}$ from 12 to 15 . This is the currently best known bound.

Proposition 5.4 If $P$ is the $9 \times 265$ lattice rectangle then $\mu(P)=15$, and hence $\phi(3) \geq$ 15.

Pfetsch and Ziegler have made extensive calculations of the numbers $\mu(P)$ for various lattice rectangles $P$. Their computational results are posted at the website

http://www.zib.de/pfetsch/chambers/

The data posted at this website seem to suggest that the answer to the question in Problem 5.2 is more likely to be negative.

The example referred to in the proposition above consists of all lattice points $(i, j)$ where $0 \leq i \leq 9$ and $0 \leq j \leq 265$. Pfetsch and Ziegler identified two chambers which are 15-gons in the unit square with vertices $(0,132),(0,133),(1,132),(1,133)$. Note that one of the 
edges of this square lies on the boundary of the $9 \times 265$ lattice rectangle. It seems that this is not a coincidence: Ernest Croot has shown that any chamber with many edges must be located close to the boundary of $P$.

\section{Virtual chambers and virtual initial ideals}

In Section 4 we established the bijection between chambers of a supernormal configuration $B$ and initial monomial ideals of $J_{B}$. In this section we will extend it to a bijection between virtual chambers of $B$ and virtual initial ideals of $J_{B}$, proving Theorem 1.2. First we define these objects and explain how the bijection works.

Throughout this section we assume that $B=\left\{b_{1}, \ldots, b_{n}\right\}$ generates the lattice $\mathbb{Z}^{m}$. This holds if $B$ is supernormal by Proposition 3.1. Under this hypothesis we can find a configuration $A=\left\{a_{1}, \ldots, a_{n}\right\} \subseteq \mathbb{Z}^{n-m}$ such that the integer kernel of the $(n-m) \times n$ matrix $\left(a_{1}, \ldots, a_{n}\right)$ is spanned by the rows of the matrix $\left(b_{1}, \ldots, b_{n}\right)$. We will also use the notation $A$ for the first matrix and $B$ for the second one. The relationship between $A$ and $B$ is called Gale duality [10, Chapter 6]. Note that the property of being supernormal is not preserved under Gale duality. It is well-known $[1,2]$ that the poset of regular subdivisions of $A$ (ordered by refinement) is antiisomorphic to the face poset of the chamber complex of $B$.

The minimal elements of the poset of regular subdivisions of $A$ are the regular triangulations of $A$ and they correspond to the full-dimensional chambers of $B$. This correspondence can be described explicitly. Let $\Delta=\left\{\sigma_{1}, \ldots, \sigma_{k}\right\}$ be the maximal cells of a regular triangulation of $A$ where $\sigma_{i}=\left\{a_{i_{1}}, \ldots, a_{i_{n-m}}\right\}$. This defines the chamber $\bigcap_{t=1}^{k}$ cone $\left(\bar{\sigma}_{t}\right)$ where $\bar{\sigma}_{i}=\left\{b_{j}: j \notin\left\{i_{1}, \ldots, i_{n-m}\right\}\right\}$. The bijection between the regular triangulations of $A$ and the maximal chambers of $B$ was extended in [3] to all triangulations of $A$.

Definition 6.1 Let $\Delta=\left\{\sigma_{1}, \ldots, \sigma_{k}\right\}$ be any (not necessarily regular) triangulation of the configuration $A$. Then the collection of complementary subsets $\left\{\bar{\sigma}_{1}, \ldots, \bar{\sigma}_{k}\right\}$ of $B$ is called a virtual chamber of $B$.

The configuration in figure 1 of the Introduction is given by the columns of the matrix

$$
\left(\begin{array}{llllll}
1 & 1 & 1 & 1 & 1 & 1 \\
0 & 1 & 2 & 0 & 1 & 2 \\
0 & 0 & 0 & 1 & 1 & 1
\end{array}\right) .
$$

This configuration $B$ has 18 virtual chambers. 16 of these are chambers and hence visible in figure 1 . The two additional virtual chambers are

$$
\begin{aligned}
& \{(1,3,4),(1,3,5),(1,4,6),(1,5,6),(2,3,4),(2,3,5),(2,4,6),(2,5,6)\} \\
& \{(1,2,5),(1,2,6),(1,3,5),(1,3,6),(2,4,5),(2,4,6),(3,4,5),(3,4,6)\}
\end{aligned}
$$

We invite the reader to "locate" these virtual chambers in figure 1 . Note that any choice of matrix $A$ must be a vector configuration in $\mathbb{Z}^{3}$. 
We define an $(n-m)$-dimensional grading of the polynomial ring $S=k\left[x_{1}, \ldots, x_{n}\right]$ by setting the degree of $x_{i}$ to be $a_{i}$ for $i=1, \ldots, n$. Thus $S$ is graded by the monoid $\mathbb{N} A$ which is spanned by the Gale dual configuration $A$. The ideal $J_{B}$ is homogeneous in this grading since

$$
x^{u}-x^{v} \in J_{B} \quad \text { if and only if } \quad \sum_{i=1}^{n} u_{i} a_{i}=\sum_{i=1}^{n} v_{i} a_{i}
$$

The Hilbert function of the quotient ring $S / J_{B}$ is given by

$$
\operatorname{dim}_{k}\left(\left(S / J_{B}\right)_{b}\right)= \begin{cases}1 & \text { if } b \in \mathbb{N} A \\ 0 & \text { otherwise }\end{cases}
$$

A homogeneous ideal in $S$ with the same Hilbert function as $J_{B}$ was called an $A$-graded ideal in [8, Section 10]. Monomial $A$-graded ideals include, but are not limited to, initial ideals of $i n_{w}\left(J_{B}\right)$.

Definition 6.2 A monomial ideal $M$ in $S$ is a virtual initial ideal of $J_{B}$ if the Hilbert function of $S / M$ is equal to the Hilbert function (5). This means that for every degree $b \in \mathbb{N} A$ there is exactly one monomial $x^{u}$ of degree $b$ with the property that $x^{u} \notin M$.

To illustrate this definition and Theorem 1.2 we compute a virtual initial ideal of $J_{B}$ for (4). First consider $w=(2,2,1)$. Then

$$
\operatorname{in}_{w}\left(J_{B}\right)=\left\langle x_{1} x_{2} x_{3}, x_{4} x_{5} x_{6}, x_{3} x_{5} x_{6}^{2}, x_{1}^{2} x_{2}-x_{5} x_{6}^{2}, x_{4}^{2} x_{5}-x_{2} x_{3}^{2}, x_{3} x_{6}-x_{1} x_{4}\right\rangle
$$

This $A$-graded ideal corresponds to the centroid in figure 1. By replacing each of the three binomials by one of its terms, we get eight virtual initial ideals of $J_{B}$, one for each virtual chamber adjacent to the centroid in figure 1. For instance, taking the first term in each of the three binomial generators of $i n_{e}\left(J_{B}\right)$ gives the virtual initial ideal

$$
\begin{aligned}
& \left\langle x_{1}^{2}, x_{3}, x_{4}\right\rangle \cap\left\langle x_{1}^{2}, x_{3}, x_{5}\right\rangle \cap\left\langle x_{1}, x_{4}^{2}, x_{6}\right\rangle \cap\left\langle x_{1}, x_{5}, x_{6}\right\rangle \\
& \cap\left\langle x_{2}, x_{3}, x_{4}\right\rangle \cap\left\langle x_{2}, x_{3}, x_{5}\right\rangle \cap\left\langle x_{2}, x_{4}^{2}, x_{6}\right\rangle \cap\left\langle x_{2}, x_{5}, x_{6}\right\rangle \cap\left\langle x_{1}^{2}, x_{3}, x_{4}^{2}, x_{6}\right\rangle .
\end{aligned}
$$

We pass to the radical of this ideal by erasing all exponents, and deleting the embedded component at the end. The eight remaining index sets are precisely the cells in the first virtual chamber listed for example (4). This process of using primary decomposition to read off the virtual chamber from a given virtual initial ideal works in general:

Remark 6.3 The map referred to in Theorem 1.2 is given by:

$$
M \mapsto\left\{\bar{\sigma}:\left\langle x_{i}: i \in \bar{\sigma}\right\rangle \text { is a minimal prime of } M\right\}
$$


This remark follows essentially from [8, Theorem 10.10]. We shall give an alternative description of the map (6) after Lemma 6.6 below. That description will be self-contained, with no reference to [8] needed, and better suited for the purpose of proving Theorem 1.2.

For arbitrary configurations $B$, the map (6) is neither injective nor surjective. Two virtual initial ideals can give rise to the same virtual chamber and there might be virtual chambers which do not correspond to virtual initial ideals [8, Theorem 10.13]. What we are claiming in Theorem 1.2 is that for supernormal configurations $B$ the map (6) is both injective and surjective. In the special case when $B$ is unimodular this was proved in [8, Lemma 10.14].

We next present a characterization of virtual monomial ideals in terms of the integral polyhedra $Q_{c}$ introduced in Section 4 .

Lemma 6.4 A monomial ideal $M$ is a virtual initial ideal of $J_{B}$ if and only if, for every $c \in \mathbb{Z}^{n}$, the polyhedron $Q_{c}$ is either empty or $Q_{c}$ contains a unique lattice point $z$ such that $\prod_{i=1}^{n} x_{i}^{c_{i}-b_{i} \cdot z}$ is not in $M$.

Proof: The map $z \mapsto \prod_{i=1}^{n} x_{i}^{c_{i}-b_{i} \cdot z}$ is a bijection between the set of lattice points in $Q_{c}$ and the set of monomials in $S$ having degree $\sum_{i=1}^{n} c_{i} a_{i}$. Hence the condition in the lemma states that every non-zero graded component of $S$ contains exactly one monomial which is not in $M$.

In [8, Proposition 10.8] it was shown that the lattice point $z$ chosen as in Lemma 6.4 need not be a vertex of the polyhedron $Q_{c}$. This is not the case for initial ideals of $J_{B}$, and the following important lemma states that it is also not the case if $Q_{c}=P_{c}$.

Lemma 6.5 If $P_{c}$ is non-empty and equal to $Q_{c}=\operatorname{conv}\left(P_{c} \cap \mathbb{Z}^{m}\right)$ then the lattice point $z$ selected in Lemma 6.4 is a vertex of $Q_{c}$.

Proof: Let $z_{1}, \ldots, z_{r}$ be the vertices of $P_{c}=Q_{c}$ and let $x^{u_{1}}, \ldots, x^{u_{r}}$ be the corresponding monomials in $S$ of degree $b=\sum_{i=1}^{n} c_{i} a_{i}$.

We first show that every monomial in $S_{r b}$ lies in the monomial ideal $\left\langle x^{u_{1}}, \ldots, x^{u_{r}}\right\rangle \subseteq S$. In polyhedral terms, if $z$ is any lattice point in $P_{r c}=Q_{r c}$, then $z$ can be written as $z=$ $\sum_{i=1}^{r} \gamma_{i} z_{i}+w$ where $w \in P_{0}$ and the $\gamma_{i}$ are non-negative reals summing to $r$. This means that for the corresponding monomial $x^{u}$ we have $u=r c-B z=r c-\sum_{i=1}^{r} \gamma_{i} B z_{i}-B w=$ $\sum_{i=1}^{r} \gamma_{i} u_{i}-B w$, where $B w \in\left(\mathbb{Z}_{\leq 0}\right)^{n}$, since $w \in P_{0}$. There exists an index $j \in\{1, \ldots, r\}$ such that $\gamma_{j} \geq 1$ and this implies that $u \geq u_{j}$, and thus that $x^{u_{j}}$ divides $x^{u}$. This shows that $S_{r b}$ lies in $\left\langle x^{u_{1}}, \ldots, x^{u_{r}}\right\rangle$.

Since our virtual initial ideal $M$ must have a standard monomial of degree $r b$, it cannot contain the ideal $\left\langle x^{u_{1}}, \ldots, x^{u_{r}}\right\rangle$, and we conclude that one of the monomials $x^{u_{j}}$ is not in $M$, as desired.

We next present an alternative characterization of triangulations of $A$, and hence of virtual chambers of $B$. A subset $U$ of the closed orthant $\mathbb{R}_{+}^{n}$ is an order ideal if $v \in U$ and $u \leq v$ coordinatewise implies $u \in U$. Let $\pi$ be the linear map $\left(\lambda_{1}, \ldots, \lambda_{n}\right) \mapsto \sum_{i=1}^{n} \lambda_{i} a_{i}$ from $\mathbb{R}_{+}^{n}$ onto cone $(A)$. A section of $\pi$ is a map $s: \operatorname{cone}(A) \rightarrow \mathbb{R}_{+}^{n}$ such that the composition $\pi \circ s$ is the identity on cone $(A)$. Note that every triangulation $\Delta$ of $A$ defines a section $s_{\Delta}$ 
as follows: $s_{\Delta}(b)$ is the unique vector $u \in \mathbb{R}_{+}^{n}$ with $A u=b$ and whose support is a cell of $\Delta$. The image $\operatorname{im}\left(s_{\Delta}\right)$ of such a section $s_{\Delta}$ is an order ideal in $\mathbb{R}_{+}^{n}$.

Lemma 6.6 The map $\Delta \mapsto s_{\Delta}$ is a bijection between triangulations of $A$ and sections $s$ of $\pi$ for which $\operatorname{im}(s)$ is an order ideal in $\mathbb{R}_{+}^{n}$.

Proof: It is clear that the section $s_{\Delta}$ associated to a triangulation $\Delta$ of $A$ satisfies the desired conditions, so we need only show that every section $s$ satisfying the hypothesis comes from a triangulation.

Fix such an $s$. We first observe that $s(r b)=r s(b)$ for $b \in \operatorname{cone}(A)$ and $r \in \mathbb{R}_{+}$. If $r<1$ then $c=r s(b) \in \operatorname{im}(s)$, and so $\pi(c)=r \pi(s(b))=r b$ satisfies $s(r b)=c=r s(b)$. The case that $r>1$ follows from this.

We claim that the set of all possible supports of vectors in $\operatorname{im}(s)$ is a triangulation of $A$. We first show that the subsets of $A$ indexed by these supports are linearly independent. Suppose not, so for some $b \in \mathbb{R}_{+}^{n}$ there is a vector $u=\left(u_{1}, \ldots, u_{n}\right)$ such that $A u=b$ where $\operatorname{supp}(u)$ is a proper subset of $\operatorname{supp}(s(b))$. There is some $r>0$ for which $r u<s(b)$, and so $r u \in \operatorname{im}(s)$. Now $\pi(r u)=r \pi(u)=r b$, so $s(r b)=r u$. This implies that $s(b)=u$, a contradiction since $\operatorname{supp}(s(b))$ properly contains $\operatorname{supp}(u)$.

This shows that the cones cone $\left(a_{i}: i \in \operatorname{supp}(s(b))\right)$ as $b$ ranges over cone $(A)$ are simplicial and that they cover cone $(A)$. We also note that this argument actually shows that for any $b^{\prime}$ in the relative interior of $\operatorname{cone}\left(a_{i}: i \in \operatorname{supp}(s(b))\right)$ we have $\operatorname{supp}\left(s\left(b^{\prime}\right)\right)=$ $\operatorname{supp}(s(b))$. Hence the relative interiors of two distinct cones do not intersect. The order ideal hypothesis guarantees that these cones form a simplicial fan.

This bijection means we can express the map in Theorem 1.2 as taking a virtual initial ideal $M$ to a section $s$ such that $\operatorname{im}(s)$ is an order ideal in $\mathbb{R}_{+}^{n}$. Fix $M$. For $P_{c}=Q_{c}$ we set $s(A c)=c-B z$ where $z$ is given by Lemma 6.5. Since $s(r b)=r s(b)$ we can extend this to all rational $P_{c}$, and hence to all $b \in \operatorname{cone}(A)$ by continuity.

Now we are ready to prove Theorem 1.2. Recall that a polyhedron $P_{c}$ is tight if and only if the greatest common divisor of all monomials of the form $x^{c-B z}$ for $z \in P_{c}$ is one. If $P_{c}$ is not tight, let $x^{w}$ be the greatest common divisor of all monomials of the corresponding degree. Then if a monomial ideal $I$ is generated in tight degrees, $x^{u} \notin I$ implies $x^{u-w} \notin I$ where $u=c-B z$ for some $z \in P_{c}$. We first present the part of the proof that holds for a general configuration.

Lemma 6.7 Let $x^{u}$ divide $x^{v}$, and let $x^{w}$ and $x^{w^{\prime}}$ be the greatest common divisors of all monomials of the same degree as $x^{u}$ and $x^{v}$ respectively. Then $x^{u-w}$ divides $x^{v-w^{\prime}}$.

Proof: Suppose this is not the case, so there is some $i$ with $(u-w)_{i}>\left(v-w^{\prime}\right)_{i}$. Since the greatest common divisor of all monomials of the same degree as $x^{u-w}$ is 1 , we know that $P_{u-w}$ is tight, and so there is some lattice point $z \in P_{u-w}$ such that $b_{i} \cdot z=(u-w)_{i}$. Because $u-w<v$, we also have $z \in P_{v}$. This means $x^{v-B z}$ is a monomial of the same degree as $x^{v}$, and is thus divisible by $x^{w^{\prime}}$, so $v-w^{\prime}-B z \geq 0$. But this implies that $b_{i} \cdot z=(u-w)_{i} \leq\left(v-w^{\prime}\right)_{i}$, a contradiction. 
Proof of Theorem 1.2: $\quad$ For each virtual chamber of $B$ we will construct a virtual initial ideal which maps to it. The construction will make it clear that this map is injective. Let $s$ be the section of $\pi$ corresponding to our virtual chamber, as described in Lemma 6.6. It is straightforward to check that $s(A c)$ is a vertex of the polyhedron $P_{c}$ for every $c \in \mathbb{R}_{+}^{n}$.

We define $M$ to be the ideal generated by all monomials $x^{c}$ such that $P_{c}$ is tight and $c$ is not in the image of $s$. We claim that $M$ is a virtual initial ideal. By construction, $M$ has at most one standard monomial in every tight degree, and thus in every degree. Tight polyhedra are integral by Theorem 3.6 and Lemma 4.4. If $P_{c}$ is tight then $s(A c)$ is a vertex of $P_{c}=Q_{c}$ and hence $s(A c) \in \mathbb{N}^{n}$. We claim $x^{s(A c)} \notin M$ for all $c$ such that $P_{c}$ is tight. If not, there is some generator $x^{v}$ of $M$ with $P_{v}$ tight dividing $x^{s(A c)}$. But since $\operatorname{im}(s)$ is an order ideal, we must have $v \in \operatorname{im}(s)$, contradicting $x^{v} \in M$. Therefore $x^{s(A c)} \notin M$.

If $P_{c}$ is not tight, let $x^{w}$ be the greatest common divisor of all monomials of degree $A c$. Then we claim that $x^{u+w} \notin M$, where $x^{u} \notin M$ satisfies $u=c-w-B z$ for $z \in P_{c-w}$. Otherwise there would be some generator $x^{v}$ of $M$ with $x^{v}$ dividing $x^{u+w}$. But since $P_{v}$ would then be a tight degree, Lemma 6.7 would imply that $x^{v}$ must divide $x^{u}$, a contradiction. This concludes the proof that $M$ is a virtual initial ideal.

The virtual initial ideal $M$ just constructed is clearly mapped back to $s$ under the map (described after Lemma 6.6) from virtual initial ideals to triangulations. Hence this map is a bijection as desired.

\section{Acknowledgment}

We thank Miguel Azaola, Ernest Croot, Jesús de Loera, Tracy Hall, Marc Pfetsch, Jörg Rambau, Francisco Santos, and Günter Ziegler for permitting us to discuss their work in Section 5 .

\section{Note}

1. After this manuscript was submitted for publication in May 2001, Tracy Hall from UC Berkeley announced a negative solution to the Polygon Problem.

\section{References}

1. L.J. Billera, P. Filliman, and B. Sturmfels, "Constructions and complexity of secondary polytopes," Advances in Mathematics 83(2) (1990), 155-179.

2. L.J. Billera, I.M. Gel'fand, and B. Sturmfels, "Duality and minors of secondary polyhedra," J. Combin. Theory Ser. B 57(2) (1993), 258-268.

3. J.A. de Loera, S. Hoşten, F. Santos, and B. Sturmfels, "The polytope of all triangulations of a point configuration," Documenta Mathematica 1(4) (1996), 103-119 (electronic).

4. S. Hoşten and R.R. Thomas, "The associated primes of initial ideals of lattice ideals," Mathematical Research Letters 6 (1999), 83-97.

5. S. Hoşten and D. Maclagan, "The vertex ideal of a lattice," Advances in Applied Mathematics 29 (2002), 521-538. 
6. M. Saito, B. Sturmfels, and N. Takayama, Gröbner Deformations of Hypergeometric Differential Equations, Springer, Heidelberg, 2000.

7. A. Schrijver, Theory of Linear and Integer Programming, John Wiley \& Sons Ltd., Chichester, 1986.

8. B. Sturmfels, Gröbner Bases and Convex Polytopes, American Mathematical Society, Providence, RI, 1996.

9. B. Sturmfels and R.R. Thomas, "Variation of cost functions in integer programming," Math. Programming 77(3, Ser. A) (1997), 357-387.

10. G. Ziegler, Lectures on Polytopes, Springer Verlag, Heidelberg, 1995. 